\title{
X-Ray Absorption by the Hot Intergalactic Medium
}

\section{Citation}

Perna, Rosalba, and Abraham Loeb. 1998. "X-Ray Absorption by the Hot Intergalactic Medium." The Astrophysical Journal 503 (2): L135-38. https://doi.org/10.1086/311544.

\section{Permanent link}

http://nrs.harvard.edu/urn-3:HUL.InstRepos:41393231

\section{Terms of Use}

This article was downloaded from Harvard University's DASH repository, and is made available under the terms and conditions applicable to Other Posted Material, as set forth at http:// nrs.harvard.edu/urn-3:HUL.InstRepos:dash.current.terms-of-use\#LAA

\section{Share Your Story}

The Harvard community has made this article openly available.

Please share how this access benefits you. Submit a story.

Accessibility 


\title{
X-RAY ABSORPTION BY THE HOT INTERGALACTIC MEDIUM

\author{
Rosalba Perna and Abraham Loeb \\ Harvard-Smithsonian Center for Astrophysics, 60 Garden Street, Cambridge, MA 02138
} \\ Received 1998 April 7; accepted 1998 June 24; published 1998 July 22
}

\begin{abstract}
The current census of observed baryons in the local universe is still missing a significant fraction of them according to standard big bang nucleosynthesis. Numerical simulations predict that most of the missing baryons are in a hot intergalactic medium, which is difficult to observe through its X-ray emission or the SunyaevZeldovich effect. We show that the next generation of X-ray satellites will be able to detect this gas through the X-ray absorption lines imprinted by its highly ionized metals on the spectrum of a background quasar. For the metallicity typically found in intracluster gas, up to $70 \%$ of the baryons produce $\mathrm{O}$ vIII absorption lines with an equivalent width $\gtrsim 0.1 \mathrm{eV}$. The spectrum of any high-redshift quasar is expected to show several such lines per unit redshift due to intervening gaseous halos of galaxy groups. These lines will be detectable at a signal-tonoise ratio of $\gtrsim 5$ after a day of integration with the future Constellation- $X$ telescope for any of the several tens of the brightest quasars across the sky.
\end{abstract}

Subject heading: X-rays: ISM

\section{INTRODUCTION}

The best estimate for the sum of the baryonic mass currently observed in stars, neutral hydrogen, and X-ray-emitting gas in clusters of galaxies (Fukugita, Hogan, \& Peebles 1998) falls short of the most likely value predicted by standard big bang nucleosynthesis (Burles \& Tytler 1998). Numerical simulations suggest that most of the missing baryons reside in a hot intergalactic medium, which is too rarefied to be detectable through its X-ray emission or the Sunyaev-Zeldovich effect (e.g., Ostriker \& Cen 1996; Theuns, Leonard, \& Efstathiou 1998). Much of this gas resides in the outskirts of galaxy groups or clusters and hence is likely to possess a similar metallicity to that observed in the X-ray emission spectrum from the cores of these systems (Mushotzky et al. 1996). In this Letter, we explore the possibility of detecting this hot intergalactic medium through the $\mathrm{X}$-ray absorption lines imprinted by its metals on the spectrum of a background quasar.

Detection of X-ray absorption lines from galaxy clusters had been suggested in the past as a means of measuring distances (Krolik \& Raymond 1988; Sarazin 1989). However, because of the limited sensitivity of X-ray instruments at that time, these early papers restricted their attention to the strong lines produced in the cores of rich clusters and hence to the unlikely superposition of a quasar behind such cores. The next generation of X-ray telescopes, manifested at their best by the successor to $A X A F$, Constellation- $X,{ }^{1}$ will achieve a much greater sensitivity than previously imagined. It is therefore the purpose of this Letter to quantify the full probability distribution of equivalent widths for the intergalactic X-ray absorption lines imprinted on the spectrum of any random quasar. Since much of the baryonic mass is currently assembled into virialized objects and since the present nonlinear mass scale is that of galaxy groups, most of the absorption lines would be produced by gas in the outskirts of these groups. In $\S 2$ we combine the Press-Schechter mass function and the singular isothermal sphere model to predict the density and temperature distribution of this gas in a standard cold dark matter cosmology with $\sigma_{8 h^{-1}}=0.5$. We used a Bardeen et al. (1986) power spectrum with a tilted primordial index of $n=0.7$. Because the power

${ }^{1}$ For more information, see http://constellation.gsfc.nasa.gov. spectrum is normalized on the cluster mass scale, our results depend only weakly on the choice of the power spectrum shape (and agree by construction with X-ray data for the temperature function of groups and clusters; see, e.g., Henry et al. 1995). We assume an $\Omega=1, \Omega_{b}=0.1, h=0.6$ cosmology throughout the discussion. In $\S 3$ we compute the corresponding statistics of the absorption lines produced by highly ionized metals in this gas. Finally, $\S 4$ summarizes the main conclusions of this work.

\section{STATISTICAL PROPERTIES OF THE GAS IN THE OUTSKIRTS OF GROUPS AND CLUSTERS OF GALAXIES}

Observations of the X-ray surface brightness of galaxy clusters are commonly fitted by the isothermal $\beta$-model. This model assumes that the gas temperature is constant and the radial profile of its mass density is given by

$$
\rho_{\text {gas }}(r, T)=\rho_{0}(T)\left[1+\left(\frac{r}{r_{c}}\right)^{2}\right]^{-3 \beta / 2},
$$

with $r_{c}$ being the core radius. Typical values for the parameters of this functional fit are clustered around $r_{c} \approx 0.25 \mathrm{Mpc}$ and $\beta \approx 2 / 3$ (Jones \& Forman 1984); for specificity, we adopt these values throughout our discussion. The central density can be matched at large radii far from the core $\left(r \gg r_{c}\right)$ to that of a singular isothermal profile, $\rho_{\text {gas }} \approx f_{\text {gas }} k T /\left(2 \pi \mu m_{p} G r^{2}\right)$, where $f_{\text {gas }}=\Omega_{b} / \Omega$ is the global mass fraction of the gas (assumed to equal the universal baryon-to-total matter density ratio), $T$ is the gas temperature, $m_{p}$ is the proton mass, and $\mu \approx 1.22$ is the mean atomic weight. Hence we set

$$
\rho_{0}(T)=\frac{f_{\mathrm{gas}} k T}{2 \pi \mu m_{p} G r_{c}^{2}} .
$$

We implicitly assume that the dark matter provides the gravitational potential needed to support the above gas distribution: $\phi=-\left(k T / \mu m_{p}\right) \ln \rho_{\text {gas }}+$ const.

At any given redshift, the comoving number density of galaxy groups or clusters with a temperature between $T$ and $T+d T$, namely $[d n(T, z) / d T] d T$, can be derived by combining 
the Press-Schechter (1974) mass function, $d n(M, z) / d M$, with the total mass-temperature relation calibrated from numerical simulations (e.g., Pen 1998):

$$
M(T, z)=M_{8}\left[\frac{T}{T_{8}(1+z)}\right]^{3 / 2},
$$

with $k T_{8}=4.9 \mathrm{keV}$ (for $\Omega=1$ ); $M_{8}=4 \pi \rho_{\text {crit }}\left(8 h^{-1} \mathrm{Mpc}\right)^{3} / 3$ is the mass inside a sphere of radius $8 h^{-1} \mathrm{Mpc}$ filled with the cosmological critical density $\rho_{\text {crit }}$. Each cluster is assigned a cutoff radius $R_{\text {cutoff }}$, which defines its total mass. Typically $R_{\text {cutoff }} \gg r_{c}$, and so $M(T, z) \approx 2 k T R_{\text {cutoff }} /\left(\mu m_{p} G\right)$. This condition results in an overdensity at the cluster boundary of order 10 .

The column density of gas particles along the line of sight to a background source that is projected at an impact parameter $b$ relative to the cluster center is given by $N_{\text {gas }}(b, T)=$ $\int_{-\infty}^{\infty} d z \rho_{\text {gas }}(r, T)$, with $r=\left(b^{2}+z^{2}\right)$. This yields

$$
N_{\text {gas }}(b, T)=N_{0}(T)\left[1+\left(b / r_{c}\right)^{2}\right]^{-1 / 2},
$$

where $N_{0}(T) \equiv \pi \rho_{0}(T) r_{c} / \mu m_{p}$. We denote by $Z(X) \equiv N_{X} / N_{\mathrm{H}}$ the fractional abundance of a heavy element $X$ relative to hydrogen and by $\Upsilon \equiv N^{i} / N_{X}$ the fraction of this element that is ionized $i$ times. In collisional equilibrium, the fractional abundance of the ion $X^{i}$ is only a function of temperature (Sarazin $\&$ Bahcall 1977), i.e., $\Upsilon=\Upsilon(T)$. We ignore photoionization by the X-ray background since we focus on the high-density environments of virialized objects (in which the highest equivalent-width lines are expected to occur) where collisional ionization dominates. The collisional ionization/recombination time is typically much shorter than the Hubble time, $t_{\mathrm{H}}$, at the temperatures $(\gtrsim 0.5 \mathrm{keV})$ and overdensities $(\gtrsim 10)$ of interest here. In particular, the collisional rate coefficients computed by Shull \& Van Steenberg (1982) imply $t_{\text {coll }} \sim 10^{15} \mathrm{~s} \ll t_{\mathrm{H}}$ for $T \sim 0.5 \mathrm{keV}$ and an overdensity $\sim 10$. This means that the condition of ionization equilibrium is satisfied throughout the entire virialized regions of the clusters considered here. Assuming a uniform metallicity, the column density of the ion $X^{i}$ is then

$$
N^{i}(b, T)=0.92 N_{\text {gas }}(b, T) Z(X) \Upsilon(T),
$$

where 0.92 is the fraction of hydrogen atoms by number.

The equivalent width of the absorption line produced by an ion column density $N^{i}$ is given by

$$
W^{i}=E \frac{\Delta v_{X}}{c} g(\tau)
$$

where

$$
\begin{aligned}
& g(\tau) \equiv \int_{-c / \Delta v_{X}}^{\infty} d y\{1-\exp [-\tau \phi(y)]\}, \\
& \phi(y)=\frac{1}{\sqrt{\pi}} \exp \left(-y^{2}\right),
\end{aligned}
$$

and

$$
\tau \equiv N^{i} \frac{2 \pi^{2} \alpha \lambda_{\mathrm{C}}^{2} f_{i}}{\left(E / m_{e} c^{2}\right)\left(\Delta v_{X} / c\right)}
$$

Here $E$ is the transition energy, $f_{i}$ is the oscillator strength for absorption, $m_{e}$ is the electron rest mass, $\lambda_{\mathrm{C}}=3.86 \times 10^{-11} \mathrm{~cm}$ is the electron Compton wavelength, and $\Delta v_{X}$ is the velocity dispersion of the element $X$. For a thermally broadened line, $\Delta v_{X}=\left[2 k T / A(X) m_{p}\right]^{1 / 2}$ (Rybicki \& Lightman 1979), where $A(X)$ is the atomic number of the element $X$.

\section{STATISTICS OF X-RAY ABSORPTION LINES}

Next we would like to find the mass fraction of the hot intergalactic gas that can be probed by an X-ray telescope of a given sensitivity. We define $W_{\min }$ to be the minimum equivalent width of an absorption line that can be resolved by the instrument. For a cluster temperature $T$, the column density $N^{i}\left(W_{\min }, T\right)$ of a given ion that would yield absorption with an equivalent width $W_{\min }$ is found by inverting equation (6). The impact parameter corresponding to this density can be found from equations (4) and (5), namely $b\left(W_{\min }, T\right)=$ $r_{c}\left\{\left[N_{0}^{i}(T) / N^{i}\left(W_{\min }, T\right)\right]^{2}-1\right\}^{1 / 2}$, where $N_{0}^{i}(T) \equiv N^{i}(b=0, T)$. Finally, the total gas mass enclosed within the cylinder defined by this impact parameter is given by $M_{\mathrm{gas}}\left(>W_{\min }, T\right)=$ $2 \pi \mu m_{p} \int_{0}^{b\left(W_{\min }, T\right)} d b b N_{\text {gas }}(b, T)$, yielding

$$
M_{\mathrm{gas}}\left(>W_{\mathrm{min}}, T\right)=2 \pi^{2} \rho_{0}(T) r_{c}^{3}\left(\left\{1+\left[\frac{b\left(W_{\min }, T\right)}{r_{c}}\right]^{2}\right\}^{1 / 2}-1\right) .
$$

The fraction of the total baryonic mass that is traced at a redshift $z$ through X-ray observations with an equivalent-width sensitivity $W_{\min }$ is then

$$
\frac{\Omega_{\mathrm{gas}}\left(>W_{\mathrm{min}}, z\right)}{\Omega_{b}}=\frac{1}{\Omega_{b} \rho_{\text {crit }}} \int d T \frac{d n(T, z)}{d T} M_{\mathrm{gas}}\left(>W_{\mathrm{min}}, T\right) .
$$

Figure 1 shows the baryonic mass fraction expressed in equation (8) at two redshifts, namely $z=0$ in the left-hand panel and $z=1$ in the right-hand panel. In both panels, the solid curve shows the result for the $\lambda=18.97 \AA$ absorption line of $\mathrm{O}$ VIII, and the dashed curve refers to the $\lambda=1.85 \AA$ transition of Fe XXV. In this calculation we have assumed that the spectral lines are only thermally broadened, since the lines are generically not saturated and so turbulent broadening has a negligible influence on their equivalent width. The fractional abundance $\Upsilon(T)$ has been computed by interpolating the data from the tables of Arnaud \& Rothenflug (1985) for O VIII and from those of Arnaud \& Raymond (1992) for Fe Xxv. We have assumed the metallicity of the gas to be $0.3 Z_{\odot}$ for iron and $0.5 Z_{\odot}$ for oxygen (see, e.g., Mushotzky et al. 1996; Gibson, Loewenstein, \& Mushotzky 1997; Fukuzawa et al. 1998) with the solar metallicity abundances taken from Anders \& Grevesse (1989).

Figure 1 (left) shows that at low redshifts, most of the collapsed baryons can be probed by an instrument with a sensitivity $\gtrsim 0.1 \mathrm{eV}$ to the $\mathrm{O}$ VIII line. The fraction is lower but still substantial at moderate reshifts, as shown in Figure 1 (right). Overall, the baryonic mass probed in absorption extends up to 1 order of magnitude above the value probed by the conventional methods of detecting X-ray emission (Fukugita et al. 1997). The O VIII line traces more baryons than the Fe XXV line, since the oxygen ion exists in the temperature regime of $0.1-1 \mathrm{keV}$, while the iron ion is abundant only at temperatures $\gtrsim 1 \mathrm{keV}$ (Arnaud \& Rothenflug 1985). The lower temperature regime is characteristic of galaxy groups (Henry et al. 1995; 


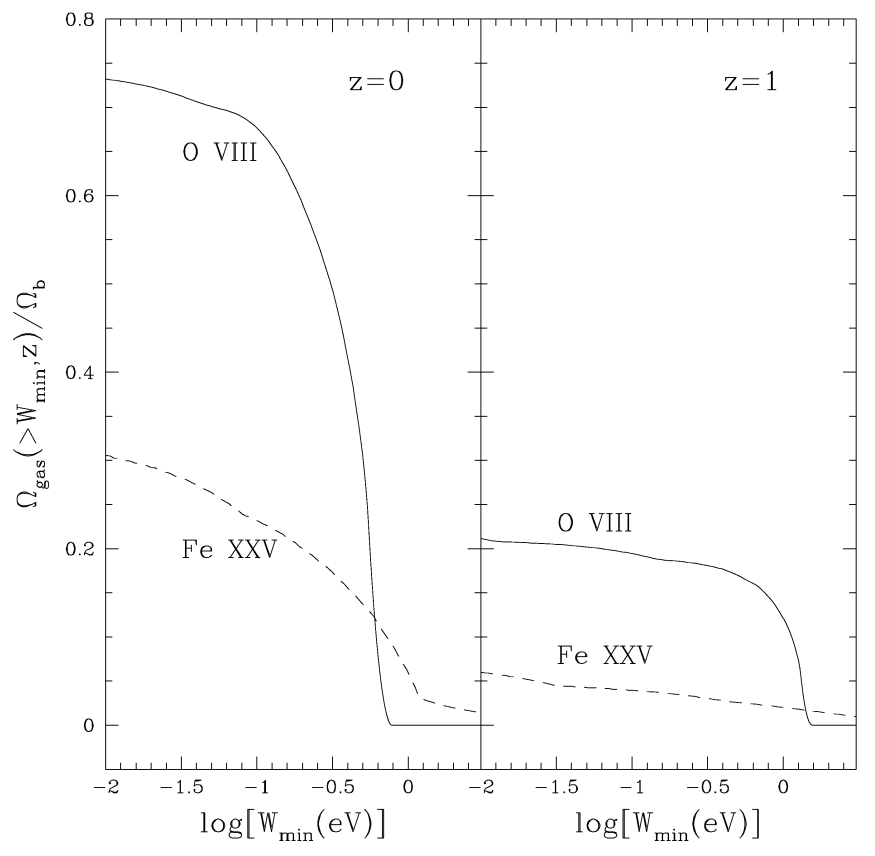

FIG. 1.-Fraction of baryons in groups or clusters of galaxies that can be probed by an X-ray telescope with an equivalent-width sensitivity $W_{\min }$ to absorption lines. The solid curve refers to absorption by the strongest resonant transition of $\mathrm{O}$ VIII [at $0.654 /(1+z) \mathrm{keV}$, where $z$ is the absorption redshift], while the dashed curve corresponds to the strongest resonant absorption by Fe Xxv [at $6.7 /(1+z) \mathrm{keV}]$.

Burns et al. 1996), which contain most of the mass in the present-day universe.

In order to assess the feasibility of these observations, it is useful to estimate the expected number of absorbers of a given equivalent width per unit redshift. The differential cross section for intercepting an ion column density between $N^{i}$ and $N^{i}+$ $d N^{i}$ within a given cluster of temperature $T$ is given by $\left[d \sigma / d N^{i}\right]\left(N^{i}\right)=2 \pi b\left(N^{i}\right)\left|d b / d N^{i}\right|_{b=b\left(N^{i}\right)}$. Using equations (4) and (5), we get

$$
\begin{aligned}
& \frac{d \sigma}{d N^{i}}\left(N^{i}, T\right) \\
& \quad= \begin{cases}2 \pi r_{c}^{2}\left[N_{0}^{i}(T)\right]^{2} /\left(N^{i}\right)^{3}, & N_{\min }^{i}(T) \leq N^{i} \leq N_{0}^{i}(T), \\
0, & \text { otherwise, }\end{cases}
\end{aligned}
$$

where $N_{\min }^{i}(T)$ is the ion column density at $b=R_{\text {cutoff }}$. Correspondingly, the differential cross section for detecting a resonant absorption line with an equivalent width between $W^{i}$ and $W^{i}+d W^{i}$ is given by $\left[d \sigma / d W^{i}\right]\left(W^{i}\right)=\left[d \sigma / d N^{i}\right]\left[N^{i}\left(W^{i}\right)\right] \times$ $\left|d N^{i} / d W^{i}\right|_{N^{i}=N^{i}\left(W^{i}\right)}$. Equation (6) then yields

$$
\begin{aligned}
\frac{d \sigma}{d W^{i}}\left(W^{i}, T\right)= & r_{c}^{2} \frac{\left[N_{0}^{i}(T)\right]^{2}}{\left[N^{i}\left(W^{i}, T\right)\right]^{3}} \\
& \times \frac{1}{\pi \alpha m_{e} c^{2} \lambda_{\mathrm{C}}^{2} f}\left[\frac{d g(\tau)}{d \tau}\right]_{\tau=\tau\left(W_{i}^{i}\right)}^{-1}
\end{aligned}
$$

for $W^{i}\left(N_{\min }^{i}\right) \leq W^{i} \leq W^{i}\left(N_{0}^{i}\right)$. Finally, by integrating over the cluster temperature distribution, we derive the probability of observing an absorption line with an equivalent width between

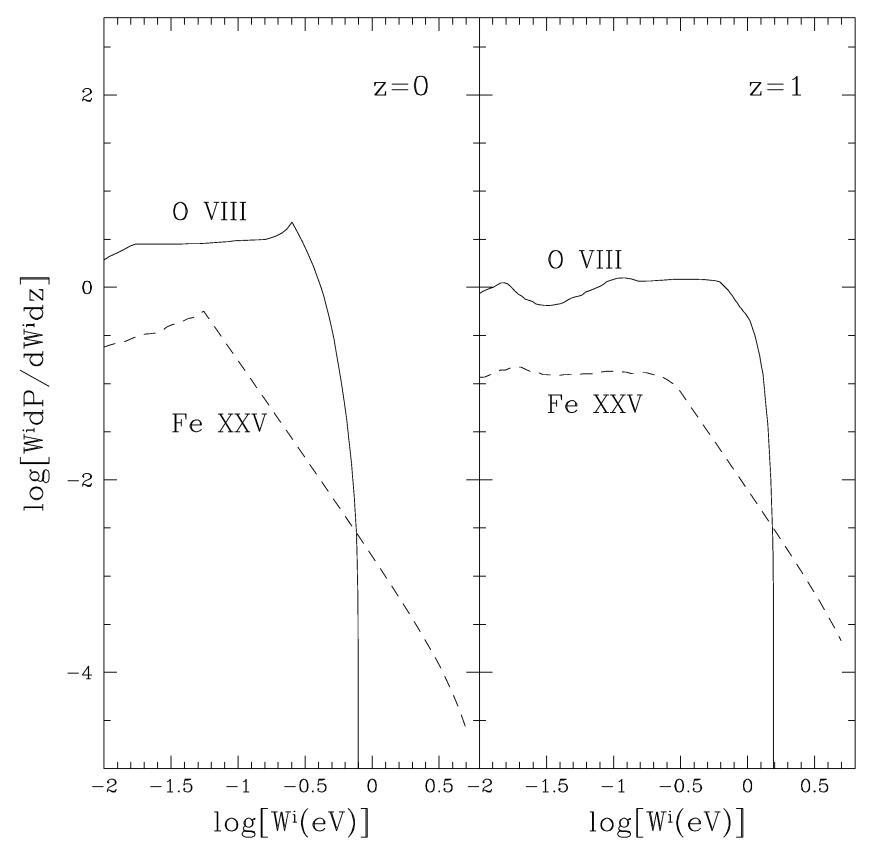

FIG. 2.-Probability for observing an absorber equivalent width $W^{i}$ per unit redshift per logarithmic equivalent-width interval as a function of the equivalent width.

$W^{i}$ and $W^{i}+d W^{i}$ per unit redshift at a redshift $z$ :

$$
\frac{d P}{d W^{i} d z}\left(W^{i}, z\right) \equiv \int d T \frac{d \sigma}{d W^{i}}\left(W^{i}, T\right) \frac{d n}{d T}(T, z)\left|c \frac{d t}{d z}\right|(1+z)^{3}
$$

where in the adopted $\Omega=1$ cosmology the redshift derivative of cosmic path length is given by $|c d t / d z|=c H_{0}^{-1}(1+z)^{-5 / 2}$, with $H_{0}$ being the Hubble constant. Figure 2 shows this probability distribution at the redshifts $z=0$ (left) and $z=1$ (right) for the previously mentioned absorption lines of $\mathrm{O}$ VIII (solid line) and Fe XxV (dashed line). We find that the spectrum of any random quasar at $z=2$ is expected to show several $\mathrm{O}$ VIII lines with an equivalent width $\gtrsim 0.1 \mathrm{eV}$ due to intervening gaseous halos of galaxy groups. New techniques for the identification of quasars behind clusters of galaxies have been recently developed (Knezek \& Bregman 1998).

Finally, we would like to estimate the detectability of the predicted absorption signal for an X-ray telescope of a given area and spectral resolution. The maximum signal-to-noise ratio $(\mathrm{S} / \mathrm{N})$ is dictated by photon counting statistics. Given the characteristic surface brightness distribution of clusters, emission by the intracluster gas can be ignored at several core radii for a characteristic telescope aperture $\leqslant 1^{\prime}$. The required number of continuum photons from the quasar that the detector must receive within its spectral resolution bin of width $\Delta E$ around the line is (Sarazin 1989)

$$
\text { (number of photons) } \gtrsim(\mathrm{S} / \mathrm{N})^{2}\left(\Delta E / W_{\min }\right)^{2} \text {. }
$$

Correspondingly, the flux from the quasar at the line energy $E$ 
needs to be

$$
F_{E} \gtrsim \frac{E(\mathrm{~S} / \mathrm{N})^{2} \Delta E}{A Q W_{\min }^{2} t},
$$

where $A$ is the collecting aperture of the telescope, $Q$ is the quantum efficiency of the detector, and $t$ is the integration time of the observation. The future Constellation- $X$ telescope will have an effective area of $Q A \approx 7670 \mathrm{~cm}^{2}$ and resolution of $\Delta E=2 \mathrm{eV}$ at the energy $E \sim 0.6 \mathrm{keV}$ (H. Tananbaum 1998, private communication). It will thus be able to detect an $\mathrm{O}$ VIII line with an equivalent width $W_{\min }=0.1 \mathrm{eV}$ at a $\mathrm{S} / \mathrm{N}$ equal to 5 after $t=10^{5} \mathrm{~s}$ of integration on a quasar with $F_{E} \gtrsim 8 \times$ $10^{-12}$ ergs $\mathrm{cm}^{-2} \mathrm{~s}^{-1} \mathrm{keV}^{-1}$. Based on the number counts of quasars brighter than a flux $S$ in the $0.5-3.5 \mathrm{keV}$ band, $N(>S) \approx 20 \times\left(S / 10^{-11} \mathrm{ergs} \mathrm{cm}^{-2} \mathrm{~s}^{-1}\right)^{-1.5} \mathrm{sr}^{-1}$ (Maccacaro et al. 1982) and the intrinsic soft X-ray spectrum of quasars $F_{E}$ $\propto E^{-1.6}$ (Laor et al. 1997), we estimate that there should be several tens of quasars above the necessary flux threshold across the sky. Figure 1 therefore implies that Constellation- $X$ will be able to probe up to $70 \%$ of the hot gas in the outskirts of groups or clusters of galaxies through the $\mathrm{O}$ VIII resonant absorption line.

\section{CONCLUSIONS}

We have investigated the possibility of using the X-ray absorption spectrum of a background quasar to probe the presence of heavy elements in the outer regions of groups and clusters of galaxies. Figure 1 demonstrates that observations of the $\mathrm{O}$ VIII line with an equivalent width sensitivity $\gtrsim 0.1 \mathrm{eV}$ should be able to probe most of the baryonic material out to the virial radius of these systems, well beyond the regions in which $\mathrm{X}$ ray emission is currently detected. We estimate that there should be several such $\mathrm{O}$ vIII absorption lines per unit redshift along the line of sight to any quasar (see Fig. 2). The future Constellation- $X$ telescope will be able to detect these lines with an $\mathrm{S} / \mathrm{N} \gtrsim 5$ in a day of integration for any of the few tens of the brightest quasars across the sky.
Our model assumed that the gas in groups or clusters of galaxies is isothermal. The modest radial decline which was observed recently with $A S C A$ for the temperature of the gas in the outer parts of clusters (Markevitch et al. 1998) might make absorption features by ionic species such as $\mathrm{O}$ VII more prominent than $\mathrm{O}$ VIII in the $\mathrm{X}$-ray absorption spectra of quasars. On the other hand, energy input by the same supernovae that enrich the gas with metals might increase the temperature of $\mathrm{X}$-ray halos beyond the value adopted in equation (3) (which was calibrated based on numerical simulations of gravitational dynamics only).

In our calculations, we have assumed a constant metallicity of the gas; a reduced metal abundance due to inefficient mixing in the outer parts of groups and clusters would obviously lower our predicted signal. If absorption will be detected for some quasars, it would be interesting to correlate the absorption-line redshift and sky position with X-ray or optical catalogs of galaxy clusters. If a group or a cluster is identified at the absorption redshift, it would be possible to infer the impact parameter $b$ and the metallicity $Z$ using equations (5) and (6). Observations of the $\mathrm{O}$ VIII line around X-ray clusters at different redshifts could trace the distribution of metals in the intergalactic medium and possibly shed light on the epoch and nature of the metal enrichment process.

Note added in manuscript.-After the completion of this work, we became aware of a parallel paper by Hellsten, Gnedin, \& Miralda-Escude (1998). This paper is complementary to our discussion in that it uses a numerical simulation instead of a semianalytical approach and focuses on the photoionized lowdensity intergalactic medium rather than on the collisionally ionized intracluster gas.

We thank Richard Mushotzky for useful discussions and Harvey Tananbaum for kindly providing details about the instrumentation of the future Constellation- $X$ satellite. This work was supported in part by the NASA grant NAG 5-7039 and by a fellowship from the university of Salerno, Italy.

\section{REFERENCES}

Anders, E., \& Grevesse, N. 1989, Geochim. Cosmochim. Acta, 53, 197

Arnaud, M., \& Raymond, J. 1992, ApJ, 398, 394

Arnaud, M., \& Rothenflug, R. 1985, A\&AS, 60, 425

Bardeen, J. M., Bond, J. R., Kaiser, N., \& Szalay, A. S. 1986, ApJ, 304, 15

Burles, S., \& Tytler, D. 1998, in Proc. Second Oak Ridge Symposium on Atomic and Nuclear Astrophysics, ed. A. Mezzacappa (Bristol: Institute of Physics), in press

Burns, J. O., et al. 1996, ApJ, 467, L49

Fukazawa, Y., et al. 1998, PASJ, in press

Fukugita, M., Hogan, C. G., \& Peebles, P. J. E. 1998, ApJ, submitted

Gibson, B. K., Loewenstein, M., \& Mushotzky, R. F. 1997, MNRAS, 290, 623

Hellsten, U. Gnedin, N. Y., Miralda-Escude, J. 1998, preprint (astro-ph/ 9804038)

Henry, J. P., et al. 1995, ApJ, 449, 422

Jones, C., \& Forman, W. 1984, ApJ, 276, 38

Knezek, P. M., \& Bregman, J. N. 1998, preprint (astro-ph/9805134)
Krolik, J. H., \& Raymond, J. C. 1988, ApJ, 335, L39

Laor, A., Fiore, F., Elvis, M., Wilkes, B. J., \& McDowell, J. C. 1997, ApJ, 477, 93

Maccacaro, T., et al. 1982, ApJ, 253, 504

Markevitch, M., Forman, W., Sarazin, C. L., \& Vikhlinin, A. 1998, ApJ, in press

Mushotzky, R. F., et al. 1996, ApJ, 466, 686

Ostriker, J. P., \& Cen, R. 1996, ApJ, 464, 27

Pen, U. L. 1998, ApJ, 498, 60

Press, W. H., \& Schechter, P. 1974, ApJ, 187, 425

Rybicki, G. B., \& Lightman, A. P. 1979, Radiative Processes in Astrophysics (Wiley: New York), 288

Sarazin, C. L. 1989, ApJ, 345, 12

Sarazin, C. L., \& Bahcall, J. N. 1977, ApJS, 34, 451

Shull, J. M., \& Van Steenberg, M. 1982, ApJ, 48, 95

Theuns, T., Leonard, A., \& Efstathiou, G. 1998, MNRAS, submitted 